Dalhousie French Studies

Dalhousie French Studies

Revue d'études littéraires du Canada atlantique

\title{
Introduction: Léon-Gontran Damas (28 mars 1912 - 22 janvier 1978), quarante-deux ans après sa " Disparition "
}

\section{Kathleen Gyssels}

Number 116, Summer 2020

Dossier spécial Léon-Gontran Damas

URI: https://id.erudit.org/iderudit/1071039ar

DOI: https://doi.org/10.7202/1071039ar

See table of contents

Publisher(s)

Department of French, Dalhousie University

ISSN

0711-8813 (print)

2562-8704 (digital)

Explore this journal

Cite this document

Gyssels, K. (2020). Introduction: Léon-Gontran Damas (28 mars 1912 - 22 janvier 1978), quarante-deux ans après sa « Disparition ». Dalhousie French Studies, (116), 3-7. https://doi.org/10.7202/1071039ar viewed online.

https://apropos.erudit.org/en/users/policy-on-use/ 


\title{
Introduction \\ Léon-Gontran Damas (28 mars 1912 - 22 janvier 1978) Quarante-deux ans après sa « Disparition »
}

\author{
Kathleen Gyssels
}

e e dossier trouve son origine aux Trois Îlets, à la Martinique, en 1992. Mon tout premier colloque aux Antilles avait été organisé par Robert Jouanny pour le Conseil International d'Études Francophones. Ce colloque reste gravé dans ma mémoire parce que les congressistes eurent l'immense plaisir d'aller parler, à l'Hôtel de Ville, avec le poètemaire de Fort-de-France. Aimé Césaire nous reçut avec sa générosité légendaire, en compagnie de Lilyan Kesteloot et de spécialistes de marque de ce qui s'appelait encore, selon l'intitulé de plusieurs communications, « études négro-africaines ». Autre souvenir : on offrit des livres à la vente, d'autres, gratuitement comme invendus. C'est là que, encouragée par le regretté Franco-Canadien Michel Tétu, je pris ses «textes colligés » sur Léon-Gontran Damas. Acte du Colloque (Paris, décembre 1988). Non seulement j'avais peu lu de Damas, mais qu'il soit ainsi livré à titre gracieux à des congressistes davantage orientés vers Senghor et Césaire, à côté d'autres romanciers antillais tels Édouard Glissant et Maryse Condé, me surprit quelque peu... car j'en dégageai la place toute relative du poète cayennais. C'est dire que sa « Disparition» (Perec) fut peu remarquée...

Un quart de siècle plus tard, à la veille du centenaire du premier Goncourt attribué à un écrivain de couleur, il convient de revisiter l'œuvre de Léon-Gontran Damas, car elle mérite réflexion dans le double sens du terme : action d'arrêter la pensée sur un objet, une personne, une idée, et phénomène par lequel les rayons lumineux, les ondes sonores, sont renvoyés dans une autre direction.

Dans ce numéro de Dalhousie French Studies, les auteurs s'engagent à ces deux opérations salutaires, car, d'une part, l'on est passé trop vite sur le « troisième homme de la Négritude ${ }^{1}{ }^{1}$ et que d'autre part, sa parole poétique parfois véhémente, émet, pour qui veut y prêter l'oreille, des sonorités troublantes, des échos détonants. Il surprend que même dans des thèses et publications académiques où il devrait figurer, son nom manque presque à l'appel (pensons parmi d'autres, au récent et brillant essai Poésie moderne et oralité des Amériques noires, où il aurait sans doute dû avoir davantage de place). Il est pourtant le plus « a-u-r-a-1 » (comme le disait Toni Morrison²) des poètes, avec Kamau Brathwaite qui vient de nous quitter et qui cite «Il est des nuits » dans son panorama des poètes de sa génération. "Limbo » ou "Limbé » (Pigments), c'est bien le concept-clé et le thème commun des deux confrères : la reconstruction d'une mémoire afro-diasporique à partir de sonorités captées depuis le Middle Passage, retranscrites sur la page dans un alphabet et langue d'emprunt, les figures de la liminalité ressuscitées dans un imaginaire transatlantique. Quoique la fortune littéraire de Damas ait été mesurée par la comparaison avec Senghor et Césaire, que l'on ait essentiellement restreint sa poésie à Pigments, le vrai Damas, au-delà de l'humour et de l'amour, de la hantise de la mort, ressurgit en 2013 à la barre de l'Assemblée Nationale. Voilà qu'une personne médiatique, justicière de premier plan, fit parler de lui et d'elle, en le citant avec bravoure lors d'un combien difficile vote pour le « Mariage pour tous ». Christiane Taubira plaide vivement pour l'adaptation de la loi du " gay marriage » et à la place de Jean Genet (son contemporain), d'André Gide (son

1 Tshitenge Lubabu, M.K. « Negritude: The Third Man after Césaire and Senghor ». En ligne.

2 Toni Morrison, dans une interview avec Christina Davis (Davis, p. 230). 
mentor), elle choisit le «third man of Négritude $»^{3}$, le poète de Black-Label. Il devient clair que la Garde des Sceaux en connaît par cœur de très longs passages qu'elle scande à la face d'une majorité masculine, blanche, hétérosexuelle ${ }^{4} . .$. Et elle revient à l'attaque le lendemain avec un poème de Névralgies, balayant tout ce qu'on a pu dire de son art : direct, « sans sophistication ».

A la veille du centenaire du Goncourt 1921 pour Batouala, Damas nous paraît taillé dans le même bois que son aîné René Maran. Lors d'une conférence pour le PEN American Conference Center, Damas salua celui-ci pour son courage, estimant qu'il était le premier qui osa montrer l'Afrique du dedans, à un moment où le colonialisme était encore dans le plein de sa force. Le courage du romancier qui révéla les nombreuses injustices dans les colonies françaises (A.E.F et A.O.F.), les violences sexuelles, la mutilation génitale et beaucoup d'autres atrocités meut aussi Damas. Anglophile, amoureux du jazz, écoutant « Strange Fruit » à l'envi, aux dires de Marc-Vincent Howlett (1970, 2019 : 26), Damas reste aux yeux de quelques critiques ${ }^{5}$, et de ses proches 1 'homme ondoyant et divers, « le poète blessé $»^{6}$. Tel Dominique Achille (fils de Louis Thomas Achille, figure pionnière dans Présence Africaine et présent au Premier Congrès des Ecrivains Noirs, en 1956, avec une communication sur les negro-spirituals), qui nous fait ici don d'un souvenir de famille, telle sa tante Isabelle Achille, éphémère première épouse de Damas, qui consigna un mot affectueux qui en dit long sur le pardon et l'absolution, qui parut dans L'Hommage à LéonGontran Damas. Incompris et sous-estimé, il compose « Vous dont les ricanements » (dans Névralgies), exemple parmi d'autres que son témoignage éminemment intimiste, échappait à la critique. Les temps n'étaient pas mûrs et son impasse était au moins double. On peut être consolé sans doute un peu qu'outre-Atlantique, il ait été mieux entendu. Damas a marqué des Africains-Américains comme Audre Lorde, voix queer, Ethelbert Miller, poète qui remercie Damas de l'avoir stimulé et d'avoir encouragé beaucoup d'artistes à Howard University $^{7}$, ainsi que des chercheurs comme Keith Q. Warner, Trinidadien qui publia le premier collectif en anglais sur son œuvre. Aux États-Unis, l'ex-professeur de lettres diasporiques finira son « chemin de Damas » en donnant des conférences et des entretiens (comme celui avec Alan Warhaftig, peu avant sa mort, que nous reprenons partiellement ici $^{8}$ ). Occulté dans la critique française et francophone, voire antillo-guyanaise, Damas a été chichement "rappelé » à part quelques colloques, à l'occasion de la mort d'Aimé Césaire, en décembre 2008 à Anvers et en 2012 (année du Centenaire de sa naissance) à Cayenne ${ }^{9}$. Il attend toujours son statut à Cayenne, son volume d'œuvre complète. Bien

3 Tshitenge Lubabu, M.K., Op. cit.

4 Gyssels, Kathleen. 2019, 2. Voir https://www.youtube.com/watch?v=OLCR2sAmVcI

5 Hamilton, Njelle W., « Re-Membering 'Body and Soul': Gender, Jazz, and Gwoka in Daniel Maximin's Lone Sun ». En ligne.

6 Kathleen Gyssels, Cristina Pelà. « Les blessures d'un poète. Entretien avec Marc-Vincent Howlett » (en ligne).

7 Ethelbert Miller, emails 26 janvier 2012, 27 novembre, 2012. Ethelbert Miller était sur le Campus de Howard University, entouré d'écrivains devenus depuis canoniques, dont Damas, précise-t-il dans un entretien dans Obsidian III. Literature in the African Diapsora 5.1. Il évoque un souvenir précis: « I remember, one day I was at work and Leon Damas telephoned and invited me to read poetry with him at the Martin Luther King, Jr. Memorial Public Library. That event took place on February 20, 1975. What I admired about Damas was how he was always reaching out to help young artists. When I started my Ascension Reading Series in 1974, its primary purpose was to provide an outlet for emerging Black poets and highlight the work of more established voices. I made this possible using my personal funds ».

8 Dans cet entretien à bâtons rompus, Damas évoque la visite d'une chercheuse, Madame Lagneau que je reconnais être Kesteloot (pourtant connue sous ce nom de jeune fille dans la recherche "négro-africaine »). Autre surprise, il compare la Diaspora noire et juive et aligne des généralisations assez déconcertantes: la migration juive serait volontaire et religieuse, maintenant la religion, la langue. Il va sans dire que la comparaison mériterait nuanciation, puisque cela a été contredit de nombreuses fois au fil de l'Histoire. Qui plus est, on retrouve cette polarisation quasi verbatim dans Le discours antillais de Glissant (1981, 29). Aux Antilles, s'alimente ainsi une guerre mémorielle qui n'est toujours pas finie dans la France (post)coloniale.

9 Voir l'appel à communication sur Potomitan: « Héritiers et héritages » (décembre 2008); suivi d'un deuxième appel en 2012: « L. G. Damas, poète patrimonial et postcolonial». 
qu'il inspire slammers et graffiteurs, musiciens (comme Guillaume Hazebrouck, présenté avec son «band» sur l'édition en ligne de ce numéro) et romanciers comme le Martiniquais Roland Brival, jazzman qui, dans Nègre de personne, s'amuse à un audacieux portrait, ou encore comme Catherine Le Pelletier avec Rhapsodie jazz pour Damas. Mais ces fictions pêchent soit par fantasme excessif, prêtant par exemple à Damas des romances avec Billie Holiday et Zora Neale Hurston, soit par un « rapatriement » aux accents « quasinationalistes », le poète étant, aux yeux de Le Pelletier, Guyanais avant d'être Martiniquais (du côté de sa mère).

Dans la critique académique, Damas n'échappa pas non plus à des contresens, que ce soit sous la plume de Nick Nesbitt qui ne le ménage pas pour ses sorties impitoyables dans Retour de Guyane (Nesbitt, 92-93), ou avant lui, Robert Serrano, qui ne lui pardonne pas d'avoir été, un temps, collaborateur à Radio Vichy (Serrano, 8). Certes, cette « Erreur », le poète la regrettera, mais l'incrimination est disproportionnée : en contre-exemple, Marguerite Donnadieu (Duras) n'avait-elle pas signé un rapport pour le Ministère des colonies $^{10}$ ?

Si un regain s'annonce avec de nouveaux ouvrages comme L.G. Damas. Les détours vers la Cité Neuve ${ }^{11}$, il reste d'autant plus difficile d'accès que ses poèmes posthumément édités ont été publiés dans un tirage limité et excessivement cher, et que ses inédits restent sous sceau.

Bref, sous peine que Damas ne reste à l'abri des regards, mettons-nous d'accord sur ce troisième " mousquetaire » de la Négritude, lui qui hérita d'un patronyme où une seule lettre le distingue des célébrissimes Dumas, père et fils. Comme le fils - Académicien qui affirma avec beaucoup d'ironie, à la façon de Damas, qu'il succédait à son père sous la coupole - et le père, panthéonisé à l'honneur d'une France se découvrant multiraciale Damas, sans être aussi prolifique qu'eux, reste celui qui tardivement devint immortel. Un siècle après son illustre prédécesseur romancier, dont la carrière alla des années 1830 à sa mort en 1870, ses écrits puisent dans les grandes heures de la décolonisation française, du règne esclavagiste à la fermeture des bagnes, des appels à l'autonomisation de la Guyane aux revendications récentes d'acceptation de «toutes les différences » (Taubira ${ }^{12}$ ) au sein de la «République une et indivisible ». Il y a, comme on le constatera à la lecture de ce numéro, bien des raisons pour que Damas reçoive encore l'attention du lecteur du XXI siècle. A la question « Qui fait l'auteur ? " ${ }^{13}$, doublée d'une autre « Que fait la critique ? », nous répondons que cette dernière seule décide de l'intronisation aussi tardive soit-elle, de cette voix à contre-chant dont le « combat politique au long cours » rejoint celui de Taubira, qui le compte parmi ses «météores» $(40,533$, et passim $)$. Le grand poète et romancier igbo Chinua Achebe (1930-2013) sut où résida le mérite de son «soul-brother » en lui dédiant «Pine Tree Speaking » :

Pine tree

lost now in the shade

of traitors decked out flamboyantly

marching back unabashed

http://www.potomitan.info/ki_nov/guiyan/damas3.php?utm_source=feedburner\&utm_medium=feed\&utm_ca mpaign $=$ Feed $\% 3 \mathrm{~A}+$ potomitan-info $+\% 28$ Potomitan.info $\% 29$

10 Duras, Marguerite et Philippe Roques. L'empire français. Paris: Gallimard, 1940.

11 Ouvrage d'Antonella Emina, 2018. Nous remercions Antonella Emina de ses conseils sur les contributions retenues dans ce numéro. Emina, experte de l'œuvre de Damas, qu'elle avait préparée pour une réédition dans la Collection « Planète libre ", s'est vu contrée à son tour par Marcel Bibas, le neveu du poète, si bien qu'il reste plus que difficile de faire redécouvrir «l'homme et l'œuvre» (titre de la seule biographie de Damas, celle de Daniel Racine, Paris : Présence Africaine, 1983).

12 Christiane Taubira, février 2013, plaidoyer pour «Le Mariage pour tous » à l'Assemblée nationale. Voir Youtube.

13 Gyssels, Kathleen, et Cristina Pelà, Op. cit. Voir également Mangeon, Philippe, « Qui fait l'auteur? ». 
to the colors

they betrayed"

Fine tree

erect and trustworthy

What school can teach me

your silent, stubborn fidelity?

(Achebe, 7)

Antwerp University

\section{OUVRAGES CITÉS}

Achebe, Chinua. « Pine Tree in Spring ». Collected Poems. Carcanet, 2005. Penguin, 2009.

Achille, Louis T. «Les Negro-Spirituals et l'expansion de la culture noire. » Présence Africaine 8/10 (956) : pp. 227-237.

Achille, Isabelle. «Hommage ». In Hommage à Léon-Gontran Damas. Paris : Présence Africaine, 1979 : pp. 142-143.

Brival, Roland. Nègre de personne. Paris: Gallimard, 2016.

Conroy-Kennedy, Ellen. « Leon Damas: Pigments and the Colonized Personality ». Black World (January 1972) : pp. 4-21.

Cornille, Jean-Louis. "Compte rendu de Emina, Antonella. Léon-Gontran Damas. Les détours vers la Cité Neuve ». Paris : L'Harmattan, 2018. Dalhousie French Studies 115 (2020), pp. 150-152.

Davis, Christina. « An Interview with Toni Morrison ». In Taylor-Guthrie, Danielle (ed.). Conversations with Toni Morrison. Jackson: Mississippi UP, 1994: pp. 223-238.

Emina, Antonella. Léon-Gontran Damas. Les détours vers la Cité neuve. Paris: L'Harmattan, 2018.

Glissant, Edouard. Le discours antillais. Paris : Seuil, 1981.

Gyssels Kathleen. «"In Search of the Talented Tenth": Léon-Gontran Damas et Albert Helman face à l'Amérind' "cambriolée" ». In Joubert, Claire (éd.). Le postcolonial comparé. Anglophonie, francophonie. Saint-Denis : Presses Universitaires de Vincennes, 2014, pp. 225-242.

https://www.cairn.info/le-postcolonial-compare--9782842924072-page-225.htm .

----. «World War II in Guianese and Caribbean Poetry: Martin Carter, Leon Damas, Kamau Brathwaite ». In Aje, Lawrence, Judith Misrahi-Barak (eds.). Re-imagining the Guyanas. Montpellier: Presses Universitaires de la Méditerranée, "Poco Pages ", 2019: pp. 113-133.

----. « Taubira : la "Dame en rose" qui porte la "torche de résine" de L.G. Damas : troubler le genre au sein de la République (afro-)péenne ». Essays in French Literature and Culture 56 (October 2019): pp. 39-56.

Gyssels, Kathleen, Cristina Pelà. " "Les blessures d'un poète rebelle". Marc-Vincent Howlett revient sur son entretien avec L.G. Damas de 1970 ». Continents Manuscrits 7 (2016). http://journals.openedition.org/coma/706.

Hamilton, Njelle W. « Re-Membering "Body and Soul": Gender, jazz, and gwoka in Daniel Maximin's Lone Sun». In Phonographic Memories: Popular Music and the Contemporary Caribbean Novel. New Jersey: Rutgers University Press, 2019, pp. 79109.

Howlett, Marc-Vincent. 1970. «Entretien avec L.G. Damas », repris dans Présence Africaine, 187/188 (1er et 2e semestres 2013) : pp. 25- 70,

Kesteloot, Lilyan. «L. G. Damas, au fil de la mémoire ». Portulan (October 2000) : pp. 231-234.

Le Pelletier, Catherine. Rhapsodie jazz pour Damas. Fort-de France : Éd. IDEM, 2012. 
Mangeon, Philippe. " Qui fait l'auteur?». Vacarme 43 (2008) : pp. 44-48.

Miller, Ethelbert. emails 26 janvier 2012, 27 novembre, 2012.

----. «Fathering Words of Loss and Love: An Interview with E. Ethelbert Miller ». Obsidian III. Literature in the African Diaspora 5.1 (Spring Summer 2004): pp. 9-24.

----. Blog: http://kalamu.com/neogriot/2017/04/11/literature-damas/.

Nesbitt, Nick. Caribbean Critique. Antillean Critical Theory from Toussaint to Césaire. Liverpool: Liverpool University Press, 2013.

Serrano, Richard. Against the Postcolonial. Francophone Writers at the End of Empire. Lanham: Lexington Books, 2005.

Taubira, Christiane. Plaidoyer pour « Le Mariage pour tous » à l'Assemblée nationale. février 2013, Youtube. Consulté le 2 avril 2020. $\mathrm{https}: / /$ www.youtube.com/watch? $\mathrm{v}=\mathrm{nV}-1 \mathrm{gzjCRwE}$

----. Mes météores. Combats politiques au long cours. Mémoires, témoignage. Paris : Flammarion, 2012.

Tétu, Michel. Léon-Gontran Damas. Acte du Colloque (Paris, décembre 1988). Paris: Présence Africaine, ACCT, 1989.

Tshitenge Lubabu, M.K. " Negritude: The Third Man after Césaire and Senghor ». The Africa Report (March 30 2012). Online. Consulté le 11 août 2012. https://www.theafricareport.com/7389/negritude-the-third-man-after-cesaire-andsenghor/.

Warner, Keith Q. Critical Perspectives on L.G. Damas. Washington: Three Continents Press, 1988.

Vetterato, Cyril. Poésie moderne et oralité des Amériques noires. Paris : Classiques Garnier, 2018. 